\title{
Etiology of Anemia, Iron Deficiency among Young Children and Strategies to Overcome
}

\author{
Uma Kiran ${ }^{1}$, Vinod Kumar C.S ${ }^{2}$, Ananya Gopalakrishnan ${ }^{3}$, N.K. Kalappanavar ${ }^{4}$, Suneeta Kalasuramath ${ }^{5}$ \\ ${ }^{1}$ Research Scholar, Bharathiar University, Coimbatore, Tamil Nadu, ${ }^{2}$ Professor, Department of Microbiology, \\ ${ }^{3}$ Intern, S.S. Institute of Medical Sciences and Research Centre, Davanagere, Karnataka, ${ }^{4}$ Professor \& Head, \\ ${ }^{5}$ Professor, Department of Physiology, S.S. Institute of Medical Sciences and Research Centre,
}

Davanagere, Karnataka

\begin{abstract}
Background: Anemia, iron deficiency, worm infestation and Helicobacter pylori infection is highly prevalent among young children population in India.

Objectives: To understand the aetiology of anaemia, iron deficiency, role of worm infestation and presence of Helicobacter pylori among young children population and to assess the impact of intervention

Method: 484 children of age 5-12 years from rural schools were enrolled for the study. Blood haemoglobin and serum ferritin were measured. H.pylori status was investigated with the 13C- Urea Breath Test. Stool specimen was assessed for the presence of parasites. 140 anemic children were selected for the intervention. They were randomly divided into seven groups of 20 children in each and named as group 1 to 7.1 as control, 2 iron supplementation, 3 treated for $H$. pylori alone, 4 treated for worms, 5 treated for $H$. pylori infection along with iron supplementation, 6 treated for worms along with iron supplementation $\& 7$ supplemented with iron along with treatment for $\mathrm{H}$. pylori and deworming (for duration and dose refer Methodology in the text).
\end{abstract}

Results: Of the 484 children, $30.4 \%$ were anemic. $18.8 \%$ children were iron deficient, of which, $50.3 \%$ were anemic. $13.2 \%$ children were positive for H.pylori and $50.0 \%$ children are infested with potentially pathogenic parasites. Intervention studies showed that the Group 7 children showed significant rise in hemoglobin and serum ferritin in comparison.

Conclusion: Childhood anemia continues to be a significant public health problem in school children and targeted intervention to iron deficiency either alone or in combination (Iron deficiency, worm infestation and H. pylori infection) can reduce the burden.

Keywords: Anemia, iron deficiency, H.pylori, Worm infestation, Intervention.

Introduction

Anemia is a leading public health issue in developing

\section{Correspondence Author:}

Dr. Suneeta Kalasuramath

Professor, Department of Physiology, S.S. Institute of

Medical Sciences and Research Centre, NH-4, Bypass

Road, Davanagere-577005, Karnataka, India

e-mail: sunivinu50@gmail.com

Mobile: 8884575021 countries. An estimated $30 \%$ of the world's population is anemic ${ }^{1}$. The global prevalence of anemia among $6-12 y$ old children in developing countries ranges from $36-77 \% \%^{2-4}$. In India the prevalence of anemia among $5-14 y$ old urban and rural is found to be in the range of 66.7 to $77 \%{ }^{5}$.

Iron Deficiency Anemia (IDA) can range from mild to severe health issues such as delayed growth and development and behavior problems among young children $^{6-8}$. Infection with $H$. pylori infection has an effect on iron absorption ${ }^{9}$. It is reported that $H$. pylori 
infection is observed in over $50-70 \%$ people in the world ${ }^{9,10}$. Moreover, IDA affects two billion people in the world. When two diseases have such a high prevalence in the population, definitely they may appear to be associated with each other. However, knowledge regarding any relation between H.pylori infection and IDA is limited ${ }^{10,11}$. Parasitic infestations caused by protozoa and helminthes continue to take their toll on mankind. The misery these parasites inflict on humans remains a major health problem worldwide ${ }^{9,12}$. Anemia, which can be mild to severe, acute or chronic, is commonly associated with parasitic infestations ${ }^{13,14}$. In view of the deleterious effects of iron deficiency anemia on overall development of children, present study was designed to assess the prevalence of anemia and iron deficiency among apparently healthy children of rural background and also to establish the impact of worm infestation on anemia and to evaluate the association between $\mathrm{H}$. pylori infection and IDA. The study also aimed to investigate the related changes before and after the treatment for $H$. pylori and deworming along with and without iron supplementation and the relationship of these changes among iron deficiency anemia in children.

\section{Methodology}

Three rural schools from Uddaghatta, Baramasamundra and Horkeravillages were selected from Jagalur taluk, Davangere district, Karnataka. Each school was named as Group A, B and C.

Body mass index of boys and girls were measured. Prevalence of anemia, iron deficiency, H. pylori infection and parasitic infestation were determined among study populations by standard tests $6,9,10,13,14$. Based on the prevalence of anemia, iron deficiency, $H$. pylori infection and parasitic infestation, a well-designed interventional program was executed.

Intervention Study: A total of 140 anemic children out of 147 anemia were selected for the intervention study and were randomly divided into seven groups. Each group comprised of 20 children.

Group 1 (20 numbers): Children were kept as a control group with no iron supplementation, no treatment for $\mathrm{H}$. pylori and with no deworming, for up to 3 months.

Group 2 (20 numbers): Iron supplementation by giving iron tablet to all the 20 children for three months ( $3 \mathrm{mg} / \mathrm{kg} /$ day - not to exceed $60 \mathrm{mg}$ daily).
Group 3 (20 numbers): Treatment for H. pylori was given for 2 weeks with no iron supplementation for 3 months. A triple drug therapy: lansoprazole $(0.6 \mathrm{mg} /$ $\mathrm{kg}$ per day, once daily), clarithromycin $(15 \mathrm{mg} / \mathrm{kg}$ per day, divided into 2 doses) and metronidazole $(20 \mathrm{mg} / \mathrm{kg}$ per day, divided into 2 doses) was administered for the period of 2 weeks.

Group 4 (20 numbers): Deworming was done by a single dose of albendazole $400 \mathrm{mg}$ was for the worms that are transmitted through contaminated soil.

Group 5 (20 numbers): Children were treated for $\mathrm{H}$. pylori infection for 2 weeks (Treatment same as Group 3 ) along with iron supplementation for 3 months (Iron supplementation same as Group 2).

Group 6 (20 numbers): Deworming (Same as Group 4) along with iron supplementation for 3 months (Iron supplementation same as Group 2).

Group 7 (20 numbers): Children were supplemented with iron for 3 months (Iron supplementation same as Group 2) along with both the treatment for H.pylori up to 2 weeks (Treatment same as Group 3) and deworming by a single dose (Same as Group 4).

The investigation for hemoglobin, serum ferritin levels, worm infestation and breath test for H.pylori was done before the intervention study and after the intervention period to assess the impact of intervention.

\section{Results}

Screening: A total of 484 children were enrolled for the study from three different schools from rural area of Davangere district. Age wise distribution of the children is given in the table- $1.26 .0 \%$ of the children were of the age group 11-12 years and $25.8 \%$ were of the age 9-10 years. Preponderance of girl child (51.2\%) was seen when compared with boys (48.8\%)

Anthropometric Measurements: Among 236 boys, $15(6.4 \%)$ were underweight, 3 (1.3\%) obese and $218(92.4 \%)$ were normal.

Out of 248 girls, 34 (13.7\%) were underweight, 11 (4.4\%) obese and $203(81.9 \%)$ girls were normal (Table 2).

Prevalence of Anemia: WHO criteria was used to categorize anemia among children of 5-12 year age group. Of the 484 children, 147 (30.4\%) were found to 
be anemic and 337 (69.6\%) were non-anemic. Among anemic children, mild anemia was found in $62(42.2 \%)$, moderate anemia in $53(36.1 \%)$ and $32(21.8 \%)$ were severely anemic. The prevalence of anemia was found to be highest in 5-6y age group (36.7\%). Among 7-8y age group $43(29.2 \%)$ were anemic and 29 (19.7\%) among $9-10 \mathrm{y}$. The anemia was more prevalent among girls (57.8\%) when compared to boys (42.2\%) (Table-3).

Prevalence of Iron-Deficiency: Not all anemic children were iron deficient and iron deficiency may occur without anemia. Out of 484 children studied 91 (18.8\%) were iron deficient. Among these 17 (5.0\%) were non-anemic and $74(50.3 \%)$ were found to be anemic (Table-4).

Prevalence of $\mathbf{H}$. pylori infection: Out of 484 children studied, $64(13.2 \%)$ children were found positive for $\mathrm{H}$. pylori infection by breath test. Among these $19(17.0 \%)$ were positive in 5-6y age group, 16 $(13.0 \%)$ in 7-8y, $17(14.0 \%)$ in 9-10y and 12 (10.0\%) in11-12y age group were found positive for $\mathrm{H}$. pylori infection (Table-5).

Association between Anemia, Iron Deficiency (ID), Iron Deficiency Anemia (IDA) and H. pylori: The prevalence of $\mathrm{H}$. pylori among non-anemic children was $36(10.7 \%)$, of which 17 were positive for both Iron Deficiency and H. pylori infection, whereas other 19 were positive only for H.pylori infection.

In mild anemic children, 6 of them were positive for H.pylori. Similarly among mild anemic children, 8 were positive for H.pylori and in severely anemic children, 14 were positive for H.pylori. The relationship among anemia, IDA and H. pylori infection is depicted in the Table 5 .

Prevalence of Parasitic Infestation: Parasitic infestation was examined in 474 children. 237 (50.0\%) were found infested with potentially pathogenic parasites. The most common parasitic seen are; Ascaris lumbricoides excreted by 44 (18.6\%) children, followed by Enterobius vermicular by 41 (17.3), Ancylostoma duodenale by 39 (16.5), Trichuris trichiura by 37
(15.6\%), Hymenolepis nana by 35 (14.8\%), Giardia lamblia by $33(13.9 \%)$ and Entamoeba histolytica excreted by 8 (3.4\%) children. The parasitic infestation was found more in girls $(53.2 \%)$ compared to boys $(46.8 \%)$. The prevalence of parasitic infestation was found highest among 5-6y age group (35.0\%), followed by $31.2 \%$ in $7-8 y$ children, $21.1 \%$ in $9-10 y$ and $12.7 \%$ was infested among 11-12y age group.

Comparison of hemoglobin and serum ferritin levels before and after the intervention program

After the intervention program 73 (52.1\%) children showed increase in the hemoglobin and serum ferritin levels which was highly significant.

Group 1: Increase in hemoglobin and serum ferritin level was seen in only one child $(5.0 \%)$ who was not supplemented with iron nor treated for $\mathrm{H}$. pylori and deworming. They were kept as control.

Group 2: Increase in hemoglobin concentration and serum ferritin was seen in $6(30.0 \%)$ children who were supplemented only with iron tablets.

Group 3: Rise in hemoglobin concentration and serum ferritin was seen in $13(65.0 \%)$ children who were treated only for H. pylori infection.

Group 4: Hemoglobin and serum ferritin level was increased in $9(45.0 \%)$ children for whom only deworming was done.

Group 5: Hemoglobin and serum ferritin concentration was raised in $15(75.0 \%)$ children who were treated for $\mathrm{H}$. pylori infection along with iron supplementation.

Group 6: Increase in hemoglobin and serum ferritin level was observed in $11(55.0 \%)$ children who were supplemented with iron tablets along with deworming.

Group 7: Hemoglobin and serum ferritin concentration was well improved in $18(90.0 \%)$ children of this group who were treated for H. pylori and deworming along with iron supplementation. 
Table 1: Age wise distribution of children among different schools

\begin{tabular}{|l|c|c|c|c|}
\hline \multirow{2}{*}{ Age (Years) } & \multicolumn{3}{|c|}{ Group } & \multirow{2}{*}{ Total Number (\%) } \\
\cline { 2 - 5 } & A Number (\%) & B Number (\%) & C Number (\%) & $112(23.1)$ \\
\hline $5-6$ & $41(23.7)$ & $33(22.4)$ & $38(23.2)$ & $121(25.0)$ \\
\hline $7-8$ & $45(26.0)$ & $35(23.8)$ & $41(25.0)$ & $125(25.8)$ \\
\hline $9-10$ & $42(24.3)$ & $40(27.2)$ & $43(26.2)$ & $126(26.0)$ \\
\hline $11-12$ & $45(26.0)$ & $39(26.5)$ & $42(25.6)$ & 484 \\
\hline Total & 173 & 147 & 164 & 4 \\
\hline
\end{tabular}

Table 2: Body mass index of the boys and girls among different schools

\begin{tabular}{|c|c|c|c|c|c|c|c|}
\hline \multirow{4}{*}{ Age (Years) } & \multicolumn{6}{|c|}{ Group } & \multirow{4}{*}{$\begin{array}{c}\text { Total } \\
\text { Number }(\%)\end{array}$} \\
\hline & \multicolumn{2}{|c|}{ Underweight } & \multicolumn{2}{|c|}{ Normal } & \multicolumn{2}{|c|}{ Obese } & \\
\hline & Boys & Girls & Boys & Girls & Boys & Girls & \\
\hline & Number (\%) & Number (\%) & Number (\%) & Number (\%) & Number (\%) & Number (\%) & \\
\hline $5-6$ & $8(15.4)$ & $12(23.7)$ & $44(84.6)$ & $48(23.7)$ & - & - & $112(23.1)$ \\
\hline $7-8$ & $5(9.3)$ & $9(26.0)$ & $49(23.8)$ & $57(26.0)$ & - & $1(26.0)$ & $121(25.0)$ \\
\hline $9-10$ & $2(3.0)$ & $8(24.3)$ & $62(27.2)$ & $47(24.3)$ & $2(26.2)$ & $4(24.3)$ & $125(25.8)$ \\
\hline $11-12$ & - & $5(26.0)$ & $63(26.5)$ & $51(26.0)$ & $1(25.6)$ & $6(26.0)$ & $126(26.0)$ \\
\hline Total & 15 & 34 & 218 & 203 & 3 & 11 & 484 \\
\hline
\end{tabular}

Table 3: Prevalence of anemia among children of different schools

\begin{tabular}{|l|c|c|c|c|c|c|c|}
\hline \multirow{3}{*}{ Age (Years) } & \multicolumn{9}{|c|}{ Anemia } & \multicolumn{3}{c|}{ Severe } \\
\cline { 2 - 8 } & \multicolumn{2}{|c|}{ Mild } & \multicolumn{2}{c|}{ Moderate } & \multicolumn{2}{c|}{ Total } \\
& \multicolumn{1}{|c|}{ Boys } & Girls & Boys & Girls & Boys & Number (\%) \\
\cline { 2 - 9 } & Number (\%) & Number (\%) & Number (\%) & Number (\%) & Number (\%) & Number (\%) \\
\hline $5-6$ & $10(35.7)$ & $14(41.2)$ & $6(84.6)$ & $12(37.5)$ & $5(38.5)$ & $7(36.8)$ & $54(36.7)$ \\
\hline $7-8$ & $12(42.9)$ & $8(23.5)$ & $5(23.8)$ & $9(28.1)$ & $4(30.8)$ & $5(26.3)$ & $43(29.2)$ \\
\hline $9-10$ & $3(10.7)$ & $7(20.6)$ & $6(27.2)$ & $5(15.6)$ & $3(23.1)$ & $5(26.3)$ & $29(19.7)$ \\
\hline $11-12$ & $3(10.7)$ & $5(14.7)$ & $4(26.5)$ & $6(18.8)$ & $1(7.7)$ & $2(10.5)$ & $21(14.3)$ \\
\hline Total & $\mathbf{1 5}$ & $\mathbf{3 4}$ & $\mathbf{2 1 8}$ & $\mathbf{2 0 3}$ & $\mathbf{3}$ & $\mathbf{1 1}$ & $\mathbf{1 4 7}$ \\
\hline
\end{tabular}

Table 4: Prevalence of Iron deficiency among children of different schools

\begin{tabular}{|c|c|c|c|c|c|}
\hline \multirow[b]{2}{*}{ Group } & \multicolumn{4}{|c|}{ Serum ferritin (SF) levels among different age group } & \multirow[b]{2}{*}{ Total } \\
\hline & $\begin{array}{c}\text { 5-6 years Number } \\
(\%)\end{array}$ & $\begin{array}{c}\text { 7-8 years Number } \\
(\%)\end{array}$ & $\begin{array}{c}\text { 9-10 years Number } \\
(\%)\end{array}$ & $\begin{array}{l}\text { 11-12 years } \\
\text { Number }(\%)\end{array}$ & \\
\hline Non-Anemic & $2(11.8)$ & $3(11.8)$ & $5(11.8)$ & $7(11.8)$ & 17 \\
\hline Mild anemic & $4(11.8)$ & $2(11.8)$ & $7(11.8)$ & $6(11.8)$ & 19 \\
\hline Moderate anemic & $8(11.8)$ & $11(11.8)$ & $7(11.8)$ & $7(11.8)$ & 33 \\
\hline Severe Anemic & $10(11.8)$ & $5(11.8)$ & $5(11.8)$ & $2(11.8)$ & 22 \\
\hline Total & $24(11.8)$ & $21(11.8)$ & $24(11.8)$ & $22(11.8)$ & 91 \\
\hline
\end{tabular}


Table 5: Association among anemia, iron deficiency, iron deficiency anemia and Helicobacter pylori infection

\begin{tabular}{|l|c|c|}
\hline Anaemic Status & Iron Deficiency & Helicobacter Pylori Positivity \\
\hline Non Anaemic $(\mathrm{n}=337)$ & $17(5.0)$ & $36(10.7)$ \\
\hline Anaemic $(\mathrm{n}=147)$ & $74(50.3)$ & $28(19)$ \\
\hline Mild $(\mathrm{n}=62)$ & $19(30.6)$ & $6(9.7)$ \\
\hline Moderate $(53)$ & $33(62.2)$ & $8(15.1)$ \\
\hline Severe $(\mathrm{n}=32)$ & $22(68.8)$ & $14(43.8)$ \\
\hline Total & $\mathbf{9 1 ( 1 8 . 8 \% )}$ & $\mathbf{6 4 ( 1 3 . 2 \% )}$ \\
\hline
\end{tabular}

\section{Discussion}

In view of the above findings, it is highly recommended that measures to reduce worm infestation including mass chemotherapy; should deserve high priority because of the known harmful effects of these worms. Worm infestation may also influence anemia to a large extent $\&$ calls for a deworming campaign along with IFA distribution in control program for anemia in children. It was found to be a strong predictor of anemia in the present study too. Stoltzfus et $\mathrm{al}^{4}$ found that $25 \%$ of all anemia, $35 \%$ of iron deficiency anemia and $73 \%$ of severe anemia was attributable to hookworm infection.

Emerging evidence seems to place H. pylori infection next to helminthiasis as a communicable cause of anemia ${ }^{17}$. Therefore, new initiatives designed to further decrease prevalence of iron deficiency and IDA in high-risk groups may need to address the eradication of $H$. pylori infection. Different epidemiological studies conducted all over the world have demonstrated an association between $H$. pylori infection and IDA ${ }^{17-18}$. In the present study the prevalence of $H$. pylori infection in IDA was $28(19 \%)$ this is in concurrence to the other studies $^{16-18}$. The prevalence of $H$. pylori was $13.2 \%$ in the present study. The rate of $H$. pylori infection was significantly higher in IDA (19\%) when compared to non IDA group (10.7\%). A significant association between H. pylori and IDA was found in children. Treatment for $H$. pylori infection showed increase in hemoglobin level in $13(65 \%)$ children without iron supplementation. H. pylori eradication therapy combined with iron administration showed increase in hemoglobin level in $15(75 \%)$ children which is more effective than iron administration alone for the treatment of IDA where only $6(30 \%)$ children showed the improvement. H. pylori infection may lead to IDA by impairing iron uptake or increasing the demand for iron ${ }^{18}$. These findings and those from the current study suggest that eradication of H. pylori infection is a promising approach for achieving long term recovery from IDA in certain children.

After deworming, 9 (45\%) children showed increase in hemoglobin level whereas deworming combined with iron supplementation showed increase in 11 (55\%) children. Children who were treated for $H$. pylroi and deworming along with iron supplementation showed a very good improvement where $18(90 \%)$ children showed rise in hemoglobin and serum ferritin concentration.

In the present study, age, education status, socioeconomic status and BMI was not significantly related with anemia. Mehta M and Kotecha et al., also reported that age is not a significant correlation of anemia ${ }^{18}$. Educational and socioeconomic status alone may not have any significant effect on anemia.

To conclude, childhood anemia continues to be a significant public health problem in school children and iron deficiency either alone or in combination (Iron deficiency, worm infestation and H.pylori infection) is the commonest nutritional cause of anemia in school children aged $5-12 \mathrm{y}$ in the community. Improvement in knowledge regarding anemia and factors associated with it and life style management can be taught through educational programs and through the media.

Acknowledgment: Authors would like to thank Block education officers and the Principal of the schools for the permission and logistic support, SSIM \& RC for the facilities, Bharathiyar University for the permission for the study.

Ethical Clearance: The research proposal was reviewed and cleared by the committee of Bharathiar University, Coimbatore. 
Source of Funding: Self funding

\section{Conflict of Interest: Nil}

\section{References}

1. Nestel, P. and Davisson, L. 2002. Anaemia, iron deficiency and iron deficiency anaemia, INACG Steering committee, USA.

2. De Maeyer, E. 1989. Preventing and controlling iron deficiency anaemia through primary health care. A guide to health administrators and programme managers. Geneva: World Health Organization.

3. Vinod Kumar, C.S., Anand Kumar, H., Sunita, V. and Kapur, I. Prevalence of anemia and worm infestation in school going girls at Gulbarga, Karnataka Indian Pediatrics 2003:40:70-72.

4. Stoltzfus, R.J., Chwaya, H.M., Tielsch, J.M., Schulze, K.J., Albonico, M. and Savioli, L. Epidemiology of iron deficiency anaemia in Zanzibari school children: the importance of hookworms. Am J Clin Nutr 1997;65(1): 153-9.

5. Stoltzfus, R.J., Dreyfuss, M.L., Chwaya, H.M., Albonico, M. 1997. Hookworm control as a strategy to prevent iron deficiency. Nutr Rev 1997; 55:223-32.

6. Bothwell, T.H., Charlton, R.W., Cook, J.D., Finch, C.A. 1979. Iron metabolism in man. Oxford, UK: Blackwell Scientific Publications.

7. World Health Organization, United Nations Children's Fund. 1996. Indicators for assessing iron deficiency and strategies for its prevention. Draft based on a WHO/UNICEF Consultation. Geneva: WHO.

8. Weiss, G. and Goodnough, L.T. Anemia of chronic disease. N Engl J Med 2005; 352:1011-23.

9. Windle, H.J., Kelleher, D. and Crabtree, J.E. Childhood Helicobacter pylori infection and growth impairment in developing countries: a vicious cycle? Pediatrics 2007;119: e754-9.
10. Wizla-Derambure, N., Michaud, L., Ategbo, S., Vincent, P., Ganga-Zandzou, S., Turck, D. and et al. Familial and community environmental risk factors for Helicobacter pylori infection in children and adolescents. J Pediatr Gastroenterol Nutr 2001;33: 58-63.

11. Dubois, S. and Kearney, D.J. Iron-deficiency anaemia and Helicobacter pylori infection: a review of the evidence. Am J Gastroenterol 2005; 100: 453-9.

12. Bini, E.J. Helicobacter pylori and iron deficiency anemia: guilty as charged? Am J Med 2001; 111: 495-7.

13. Malfertheiner, P., Megraud, F., O'Morain, C., Bazzoli, F., El-Omar, E., Graham, D., Hunt, R., Rokkas, T., Vakil, N. and Kuipers, E.J. Current concepts in the management of Helicobacter pylori infection: the Maastricht III Consensus Report. Gut 2007;56: 772-81.

14. Vidyarthi, S.C. Prevalence of intestinal parasites in Lucknow. Indian Journal of Medical Science 1969;23: 654-660.

15. Watkin, N.E. and Pollitt, E. "Stupidity or worms": do intestinal worms impair mental performance? Psycol Bull 1997; 121: 171-91.

16. Alam, M., Khaltak, A.L. and Talha, M. Anaemia and intestinal parasitic infestations in school children in Skardu. Pak Armed Forces Med J 2007;57: 77 - 81.

17. Cardenas, V.M., Mulla, Z.D., Ortiz, M. and Graham, D.Y. Iron deficiency and Helicobacter pylori infection in the United States. Am J Epidemiol 2006;163(2): 127-34.

18. Mehta, M. Effectiveness of daily \& weekly iron and folic acid supplementation in anaemic adolescent girls. UNICEF funded final report of the research project, Bombay urban ICDS Project 1998;21-5. 